\title{
LE DÉFI DE LA SÉCURITÉ ENVIRONNEMENTALE À L'ONU
}

Lucile Maertens

\section{François Gemenne, L'Enjeu mondial}

Presses de Sciences Po (P.F.N.S.P.) | «Annuels »

2015 | pages 205 à 214

ISBN 9782724617504

Article disponible en ligne à l'adresse :

https://www.cairn.info/l-enjeu-mondial--9782724617504-page-205.htm

\section{Pour citer cet article :}

Lucile Maertens, «Le défi de la sécurité environnementale à l'ONU », in François Gemenne, L'Enjeu mondial, Presses de Sciences Po (P.F.N.S.P.) «Annuels », 2015 (), p. 205-214.

Distribution électronique Cairn.info pour Presses de Sciences Po (P.F.N.S.P.).

(C) Presses de Sciences Po (P.F.N.S.P.). Tous droits réservés pour tous pays.

La reproduction ou représentation de cet article, notamment par photocopie, n'est autorisée que dans les limites des conditions générales d'utilisation du site ou, le cas échéant, des conditions générales de la licence souscrite par votre établissement. Toute autre reproduction ou représentation, en tout ou partie, sous quelque forme et de quelque manière que ce soit, est interdite sauf accord préalable et écrit de l'éditeur, en dehors des cas prévus par la législation en vigueur en France. Il est précisé que son stockage dans une base de données est également interdit. 


\section{Le défi de la sécurité environnementale à I'ONU}

\section{Lucile Maertens}

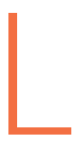

e deuxième volume du cinquième rapport du Groupe intergouvernemental d'experts sur l'évolution du climat (GIEC) consacre, pour la première fois en 2014, un chapitre intégral à la sécurité humaine. Alors que l'abondance des études recensées dans ce rapport montre l'intérêt considérable que suscite la question des impacts sécuritaires du changement climatique, le rapprochement entre problématiques environnementales et sécurité n'en est pas moins relativement récent.

Dans les études stratégiques, la définition traditionnelle de la sécurité n’a trait qu'aux menaces militaires. Cependant, le regain d'intérêt observé chez les internationalistes depuis les années 1980, et surtout depuis la fin de la guerre froide et la recomposition des grands enjeux sécuritaires dans un monde post-bipolaire, a conduit à un élargissement progressif de la notion de sécurité amorcé tant par les théoriciens (en particulier ceux de l'École de Copenhague et les tenants des théories critiques) que par les praticiens. Le sujet de la sécurité - l'entité qui bénéficie de cette sécurité - ne se limite plus à l'État (on pense notamment au concept de sécurité humaine), pas plus que l'objet de la sécurité - la menace qui met en péril la sécurité - ne se borne aux enjeux politico-militaires. Les questions environnementales telles que le changement climatique, la dégradation de l'environnement ou les catastrophes naturelles, étudiées en tant que déclencheurs potentiels de conflits ou comme menaces à la sécurité humaine, contribuent à cet élargissement et à l'approfondissement de la notion de sécurité.

Sa définition classique telle qu'elle apparaît dans la charte des Nations unies, organisation en charge de la gestion de la sécurité internationale, est d'ailleurs 
contestée par le secteur de l'environnement. Conçue après la seconde guerre mondiale, l'ONU a été structurée selon une division thématique du travail, chaque agence ou programme étant en charge d'une seule question. Or cette structure entrave le traitement de problématiques transversales telles que la sécurité environnementale qui porte sur les rivalités classiques entre deux parties en conflit (États, mais aussi communautés, villes, tribus, etc.) à la suite de la dégradation de leur environnement, sur la protection des hommes face aux menaces environnementales d'origines naturelles (catastrophes naturelles) ou humaines, ou encore sur la préservation de l'environnement (en particulier en cas de conflits).

Pourtant, l'ONU s'intéresse à l'environnement et aux questions de sécurité depuis les années 1980. Une branche spécifique du Programme des Nations unies pour l'environnement (PNUE) s'interroge sur les liens entre ressources naturelles et conflits depuis ses premières interventions au Kosovo à la fin des années 1990. Les départements des opérations de maintien de la paix (DOMP) et d'appui aux missions (DAM) tentent, quant à eux, de réduire l'empreinte écologique de leurs missions, et de nombreux programmes onusiens établissent des liens directs et indirects entre les deux questions dans leurs publications et projets. Notre objectif ici sera de montrer comment l'ONU travaille sur les questions transversales complexes de sécurité et d'environnement.

À partir des conclusions tirées d'une recherche qualitative portant sur le travail onusien - analyse de la littérature grise, observation participante et entretiens -, ce chapitre met ainsi en évidence la trajectoire du concept de sécurité environnementale au sein de l'organisation, et dévoile comment les champs de la sécurité internationale et des politiques environnementales se rencontrent à l'ONU à travers les processus de sécurisation de l'environnement, d'une part, et ceux d'« environnementalisation » de la sécurité, d'autre part. Nous commencerons par analyser la construction de l'environnement en tant qu'enjeu de sécurité dans le discours et dans les pratiques des institutions onusiennes, puis nous montrerons comment la sécurité est devenue un sujet d'intérêt pour les politiques environnementales, à travers, notamment, l'empreinte écologique des activités militaires.

\section{La construction de la menace environnementale à l'ONU}

Les travaux de l'École de Copenhague ont fondamentalement remis en cause la perception classique de la sécurité en montrant qu'il s'agit avant tout d'une construction sociale à travers ce qu'ils nomment le processus de sécurisation. Pour Barry Buzan, Ole Wæver et Jaap de Wilde (1998; Wæver, 1995), la 
sécurisation consiste, pour les élites politiques, à désigner une menace pour la survie et à la faire reconnaître comme telle (intersubjectivité) afin de pouvoir y répondre par des mesures urgentes et exceptionnelles propres au champ de la sécurité. Par exemple, si le président des États-Unis désigne les migrants comme un enjeu de sécurité national, il peut, une fois cette désignation acceptée par la société civile et le Congrès, établir un certain nombre de lois reconnaissant les migrants comme des menaces. La sécurisation procéderait donc d'un acte de langage.

Bien que fortement critiquée, notamment pour sa définition restreinte au discours, et complétée par des approches plus sociologiques que nous présenterons rapidement par la suite, cette théorie a inspiré de nombreux travaux sur la construction de l'environnement en tant qu'enjeu de sécurité. Dans la lignée des recherches de Rita Floyd (2010) sur la sécurisation environnementale par l'administration américaine et de celles de Maria-Julia Trombetta (2008) sur le discours sécurisant le changement climatique, nous analysons cette construction dans le discours onusien.

Alors que les premiers rapprochements entre problématiques environnementales et sécurité touchaient à la protection de l'environnement en cas de conflits - publications conjointes du PNUE et du Stockholm International Peace Research Institute (SIPRI) entre 1984 et 1988 -, intervention du PNUE dans les Balkans à la suite des bombardements de l'OTAN pour évaluer l'état de l'environnement -, on observe dès la fin des années 1980 la création et la diffusion du concept de sécurité environnementale par l'ONU. Les deux types de sécurité - la sécurité classique et la sécurité humaine - sont constamment mentionnés dans la construction de l'environnement comme menace à la stabilité étatique et à la vie humaine. Ainsi, le rapport Brundtland Notre avenir à tous de 1987, l'Agenda pour la paix du secrétaire général Boutros Boutros-Ghali de 1992, et le rapport du millénaire du secrétaire général Kofi Annan «Nous les peuples »: le rôle des Nations unies au XXI" siècle de 2000 établissent tous un lien clair entre environnement, tensions politiques et conflits. De même, le rapport du Programme des Nations unies pour le développement (PNUD) sur le développement humain en 1994, à l'origine du concept de sécurité humaine, définit la «sécurité environnementale » comme l'une des sept dimensions de cette nouvelle notion.

À la suite de ces premiers rapports, le PNUE s'investit largement dans la construction de l'environnement comme menace à la stabilité étatique. Dans le cadre de son sous-programme "Désastres et conflits», et surtout de sa branche «Post-conflit et gestion des désastres », il contribue activement à la sécurisation de l'environnement à l'ONU en produisant une série de rapports 
sur le lien entre environnement et conflit. S'appuyant sur un panel d'experts en matière d'environnement, de conflit et de consolidation de la paix qu'il a lui-même mis en place en 2008, et justifiant ainsi son action dans ce nouveau domaine, le programme onusien s'est lancé dans la rédaction de quatre rapports sur ce thème, dont trois ont déjà été publiés : le premier sur le cadre légal de la protection de l'environnement en cas de conflit (Protecting the Environment during Armed Conflicts: An Inventory and Analysis of International Law, 2009) ; le deuxième sur les liens entre conflit et ressources naturelles ( $D$ u conflit à la consolidation de la paix. Le rôle des ressources naturelles et de l'environnement, 2009); le dernier sur l'environnement et les opérations de maintien de la paix onusiennes (Greening the Blue Helmets: Environment, Natural Resources and UN Peacekeeping Operations, 2012). Le quatrième rapport, toujours à l'état de brouillon, doit être consacré à la diplomatie environnementale. Accordant une attention particulière aux expressions choisies, ces rapports ont contribué à sécuriser l'environnement en le présentant comme un risque pour la consolidation de la paix et de reprise des conflits, comme un enjeu de convoitise et de tensions mais aussi comme une source de financement majeure facilitant le déclenchement et la poursuite des efforts de guerre.

Enfin, le PNUE, à travers une grande variété de publications, dont les rapports du secrétaire général, facilite l'intégration des questions de sécurité et d'environnement auprès d'autres acteurs onusiens (phénomène de mainstreaming) et l'énonciation d'un discours construisant l'environnement comme un enjeu de sécurité au sein de l'ensemble de l'organisation. On retrouve aussi ce type de rapprochement dans les publications d'autres programmes tels que l'Organisation des Nations unies pour l'alimentation et l'agriculture (FAO), le Haut Commissariat pour les réfugiés (HCR) ou encore le PNUD. Le discours s'oriente autour de trois grands thèmes : les ressources naturelles et la dégradation environnementale comme source de conflits et d'insécurité ; les ressources naturelles comme source de financement de la guerre ; le changement climatique comme multiplicateur de menaces.

\section{Les pratiques de sécurisation du PNUE}

Comme nous l'avons souligné, le modèle de l'École de Copenhague centré sur une analyse discursive de la sécurisation a été largement critiqué puis enrichi. Qu'il s'agisse de proposer une étude plus complexe du discours (Stritzel, 2007), de s'intéresser plus spécifiquement aux acteurs de la sécurisation (Bigo, 2005) et à leurs intentions (Floyd, 2010) ou d'inviter à observer les pratiques de sécurisation (Balzacq, 2010), plusieurs auteurs ont défini des modèles 
complémentaires utiles pour comprendre le cas onusien et analyser comment l'ONU sécurise concrètement l'environnement.

Le PNUE y participe par la production de rapports mais également par des activités de terrain qu'il conduit en partenariat avec de nombreux acteurs onusiens - l'Office pour la coordination des affaires humanitaires de l'ONU (OCHA) concernant les urgences environnementales (désastres écologiques d'origine naturelle et humaine), le PNUD et son bureau « prévention des crises et relèvement », le Bureau d'appui à la consolidation de la paix (BACP) sur les ressources naturelles et la consolidation de la paix ou encore ONU-Femmes et extra-onusiens - notamment l'Union européenne dans le cadre d'un partenariat ONU-UE sur les ressources naturelles et la prévention des conflits, ou encore l'OTAN qui intervient au sein de l'Initiative EnvSec (Environmental Security) sur la sécurité environnementale en Asie centrale, en Europe de l'Est, dans le Caucase du Sud et les Balkans, dont le PNUE et le PNUD sont tous deux membres. Ceci confirme les conclusions de Trombetta (2008) sur le développement de nouvelles pratiques de sécurité tournées vers la gestion quotidienne du risque plutôt que vers des politiques exceptionnelles d'urgence censées, selon les auteurs de l'École de Copenhague, caractériser le secteur de la sécurité. Nous avons nous-même observé au PNUE un travail quotidien de rapprochement des problématiques environnementales et de sécurité via des pratiques d'intervention technicisées.

Les pratiques de sécurisation du PNUE reposent principalement sur deux types d'activités. Tout d'abord, la branche post-conflit et gestion des désastres produit des évaluations environnementales dans les régions en situation de conflit ou post-conflit. Le PNUE intervient également dans le cadre de l'unité jointe PNUE-OCHA et de l'initiative EnvSec, à laquelle il a pris part avec d'autres programmes onusiens et partenaires externes. Dans ce cadre, il envoie une équipe d'experts qui évalue l'état de l'environnement et identifie les risques et les opportunités, afin de mettre en œuvre d'éventuels projets d'action à plus long terme. En complément, le PNUE élabore une offre de formations et produit des directives à destination des acteurs onusiens et d'acteurs politiques locaux. Il délivre des formations aux autorités locales, afin de les sensibiliser aux enjeux de sécurité, d'environnement et de gestion des risques, et publie des fiches d'information - telles que celles produites sur l'environnement et la consolidation de la paix dans le cadre d'un partenariat entre l'ONU et l'Union européenne dont le PNUE est un membre très actif - et des cours en ligne libres d'accès - comme celui créé avec l'Institut des Nations unies pour la formation et la recherche (UNITAR) sur la base des conclusions de son étude sur l'environnement et le maintien de la paix. 
Ses intentions sont doubles. L'approche technique lui permet à la fois de respecter le mandat qui lui a été confié en tant qu'organisation normative dont la valeur ajoutée se mesure à son expertise, et d'accaparer ce champ d'action en se positionnant comme l'expert de ce domaine. Il s'agit, par ailleurs, d'une décision politique de contournement qui permet d'éviter la surpolitisation des enjeux environnementaux et les blocages souverainistes des États. C'est en faisant valoir leur statut de scientifiques «neutres » et en proposant des évaluations «impartiales» que les fonctionnaires du PNUE peuvent intervenir en situation post-conflit et dans des zones de grandes tensions politiques.

\section{L'environnement au Conseil de sécurité}

À plusieurs reprises depuis les années 2000, différents volets des problématiques environnementales ont été inscrits à l'agenda du Conseil de sécurité : ressources naturelles et conflits, environnement et maintien de la paix, changement climatique et sécurité.

Concernant les ressources naturelles, aucune résolution intégralement dédiée à cette question n'a été votée, mais on peut souligner deux résolutions du Conseil (S/RES/1459, 28 janvier 2003 ; S/RES/1625, 14 septembre 2005) et une déclaration du président (S/PRST/2007/22, 25 juin 2007) conclue à la suite d'un débat organisé en 2007 par la présidence belge sur le thème « Maintien de la paix et de la sécurité internationales : ressources naturelles et conflit ». Cette dernière reconnaît le rôle et l'importance des ressources naturelles en matière de conflits et de prévention des conflits, qui ont de nouveau été débattus au Conseil en 2013. À la suite de diverses résolutions portant sur le commerce illicite de diamants bruts, source de financement de certains conflits, le Conseil a également apporté son soutien au système de certification entré en vigueur en 2003 (processus de Kimberley). L'absence de réponse systématique et une approche au cas par cas caractérisent, in fine, la stratégie du Conseil dans le domaine des ressources naturelles et de l'environnement. Depuis 1990, plusieurs opérations de maintien de la paix ont ainsi disposé de mandats directs ou indirects pour traiter des ressources naturelles.

Enfin, de manière un peu plus polémique, le changement climatique a récemment été mis à l'agenda du Conseil. En 2007, sous la présidence britannique, il a examiné pour la première fois les possibles conséquences du changement climatique sur la sécurité internationale. Aucun accord n'est sorti de cette discussion qui a cristallisé les oppositions Nord-Sud, lesquelles se sont à nouveau manifestées en 2011 lors d'un deuxième débat organisé à la demande de la présidence allemande. Bien que le G77 et la Chine reconnaissent un lien 


\section{Dimensions environnementales des opérations de maintien de la paix de l'ONU jusqu'en 2013}

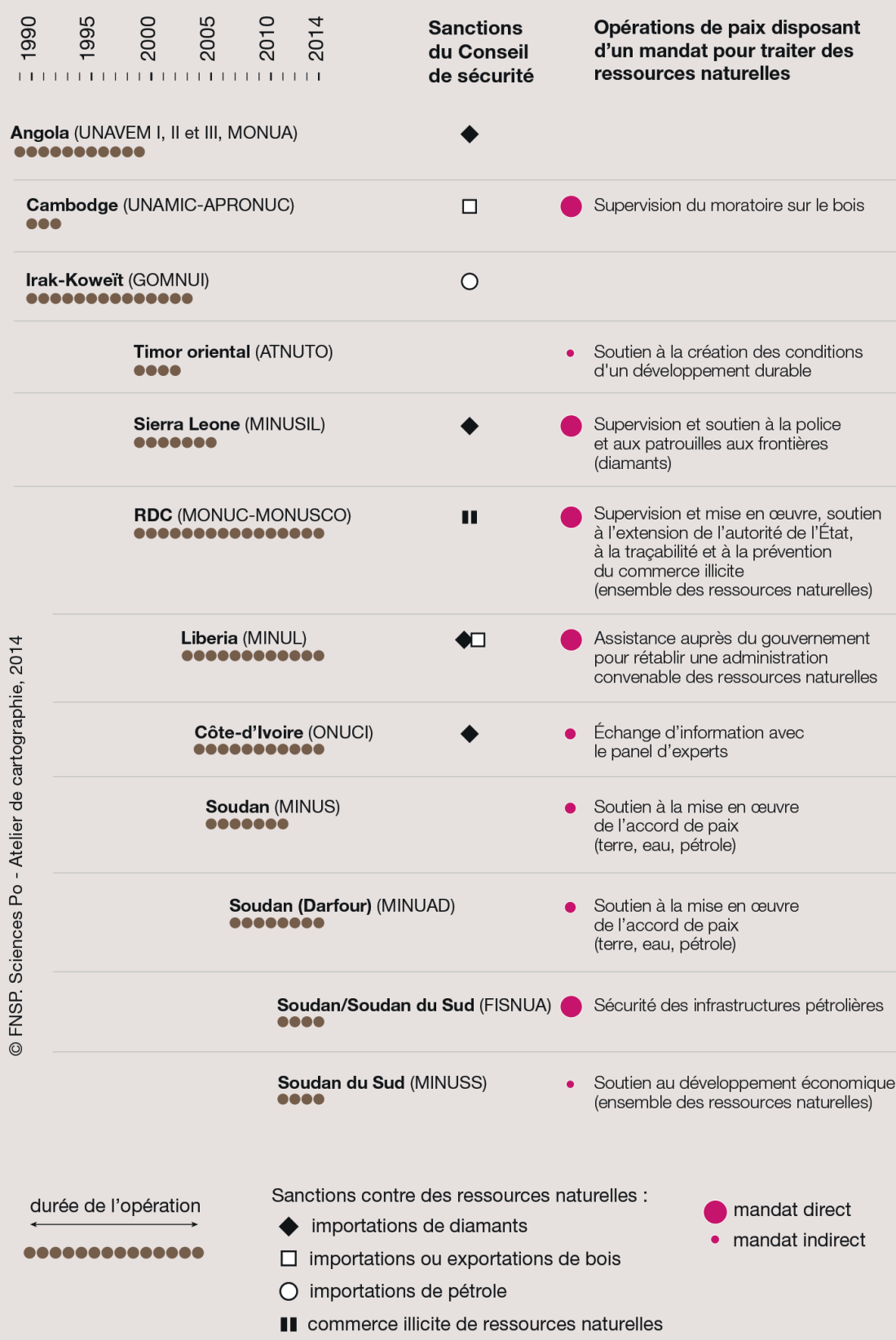

Source : Programme des Nations unies pour l'environnement (PNUE), Greening the Blue Helmets, Environment, Natural Resources and UN Peacekeeping Operations, 2012, www.unep.org 
possible entre changement climatique et sécurité, ils demandent que cela soit débattu à l'Assemblée générale - une arène universelle contrairement au Conseil de sécurité -, le changement climatique étant un enjeu de développement qui concerne tous les États. Une déclaration du président a tout de même été adoptée priant le secrétaire général d'intégrer ces éléments de contexte dans ses rapports à destination du Conseil (S/PRST/2011/15, 20 juillet 2011). Enfin, début 2013, une rencontre dans le cadre de la formule Arria rencontres informelles et confidentielles du Conseil où des acteurs externes peuvent être invités à s'exprimer sur un sujet - organisée par le Pakistan et le Royaume-Uni, a été consacrée aux implications du changement climatique sur la sécurité internationale. Même si le G77 et la Chine ont à nouveau refusé que le changement climatique soit inscrit dans les prérogatives du Conseil, on peut présumer que des actions complémentaires pourront être mises en œuvre à l'avenir, comme cela a déjà été le cas, notamment dans le cadre des opérations de maintien de la paix.

\section{" Environnementaliser » pour sécuriser}

Dans la lignée de l'étude d'Angela Oels sur la «climatisation du champ de la sécurité » (Oels, 2012), analysons maintenant les manœuvres « environnementalisant » la sécurité de l'ONU, autrement dit la construction de la sécurité comme enjeu environnemental. Ce processus s'inscrit dans la stratégie globale des Nations unies Greening the Blue, initiée en 2010. Soutenue par le secrétaire général Ban Ki-moon, elle aspire à réduire l'empreinte écologique des activités de l'organisation. Cette politique fait directement écho aux réalités du terrain, et plus précisément aux préoccupations environnementales croissantes notamment en matière d'opérations de maintien de la paix, activité la plus onéreuse et la plus visible du travail de l'ONU. Depuis la complexification des missions dans les années 1990, évoluant sur les plans quantitatif et qualitatif, leurs conséquences sur l'environnement font l'objet d'une attention croissante. En 2009, une politique environnementale a été approuvée (document interne), et des officiers environnementaux nommés dans certaines missions. L'application des directives reste toutefois inégale. Ainsi, lorsque l'épidémie de choléra survenue en Haïti à la suite du tremblement de terre de 2010 a été attribuée aux casques bleus et à leur mauvaise gestion des eaux usées (le secrétariat de l'ONU ne reconnaissant qu'une part de responsabilité limitée, le discours officiel étant qu'il s'agissait d'une cause parmi d'autres), le département des opérations de maintien de la paix et le département d'appui aux missions ont été amenés à considérer les impacts des missions sur leur environnement, en termes de 
risques écologiques et sanitaires notamment, avec encore plus d'acuité, et à les inscrire progressivement à l'agenda environnemental de l'ONU.

Cette « environnementalisation » s'avère d'autant plus intéressante qu'elle permet la mise sur agenda de la sécurité environnementale. En effet, de même que la technicisation constitue une manœuvre pertinente pour sécuriser l'environnement en évitant la surpolitisation et les enjeux de souveraineté qui font souvent obstacle à la coopération, l'« environnementalisation » de la sécurité peut soutenir la sécurisation de l'environnement. Cette manœuvre est particulièrement nette dans le rapport du PNUE sur l'environnement et le maintien de la paix intitulé Greening the Blue Helmets (2012). Ce rapport est divisé en deux parties, présentées comme logiquement connectées alors que, sur le terrain, elles ne concernent pas les mêmes problématiques et acteurs ni ne requièrent les mêmes types de solution. La première partie est consacrée à l'empreinte écologique des missions alors que la seconde touche aux ressources naturelles, aux conflits et au maintien de la paix. Cette association singulière est en réalité un choix stratégique. Dans la mesure où les fortes divisions qui règnent au sein du Conseil de sécurité n'auraient pas permis de publier un rapport entièrement consacré aux conséquences des problématiques environnementales sur la sécurité internationale et sur le maintien de la paix, le PNUE a choisi de combiner cette question à un sujet moins polémique, à savoir l'impact écologique des missions. L'« environnementalisation » de la sécurité était donc un moyen de sécuriser l'environnement.

\section{Complémentarité des actions autour de la sécurité environnementale à l'ONU}

Même si ces processus connaissent des limites, comme en témoignent les lenteurs et oppositions au sein du Conseil de sécurité, ils pourraient in fine constituer des stratégies permettant la réalisation du mandat originel de l'ONU en matière de paix et de sécurité internationales (l'environnement n'est pas mentionné dans la charte des Nations unies et sa protection n'est venue que tardivement dans les missions de l'organisation). Ainsi, l'utilisation croissante de l'environnement comme ressource pour l'action diplomatique onusienne en matière de consolidation de la paix et de prévention des conflits tend à confirmer cette dynamique. L'organisation d'une rencontre entre acteurs locaux des deux parties en conflit au Sahara occidental, l'étude des tensions entre Haïti et la République dominicaine autour des ressources forestières et les interventions en Asie centrale pour permettre la coopération en matière d'eaux transfrontalières révèlent chacune comment l'environnement constitue une 
ressource politique pour le renouvellement de l'action onusienne. Mise en péril par le développement des diplomaties de club et des organisations régionales, l'ONU utilise son mandat large et universel - contrairement à certaines de ces institutions - pour investir ce nouveau champ et freiner sa monopolisation par certaines organisations telles que l'OTAN.

\section{Pour en savoir plus .}

BALZACQ (T.) (ed.) (2011), Securitization Theory: How Security Problems Emerge and Dissolve, Londres, Routledge.

BARNETT (M.) et FINNEMORE (M.) (2004), Rules for the World: International Organizations in Global Politics, Ithaca (N. Y.), Cornell University Press.

BIGO (D.) (2005), «La mondialisation de l'(in)sécurité ? Réflexions sur le champ des professionnels de la gestion des inquiétudes et analytique de la transnationalisation des processus d'(in)sécurisation », Cultures \& Conflits, 58, p. 53-101.

BUZAN (B.), WAVVER (O.) et DE WILDE (J.) (1998), Security: A New Framework for Analysis, Boulder (Colo.), Rienner.

FLOYD (R.) (2010), Security and the Environment: Securitisation Theory and US Environmental Security Policy, Cambridge, Cambridge University Press.

OELS (A.) (2012), «From "Securitization" of Climate Change to "Climatization" of the Security Field: Comparing Three Theoretical Perspectives», dans J. Scheffran et al. (eds), Climate Change, Human Security and Violent Conflict, Heidelberg, Springer, p. 185-205.

STRITZEL (H.) (2007), «Towards a Theory of Securitization: Copenhagen and beyond», European Journal of International Relations, 13 (3), p. 357-383.

TROMBETTA (M. J.) (2008), «Environmental Security and Climate Change : Analyzing the Discourse », Cambridge Review of International Affairs, 21 (4), p. 585-602.

WFVVER (O.) (1995), «Securitization and Desecuritization », dans R. Lipschutz (ed.), On Security, New York (N. Y.), Columbia University Press, 1995, p. 46-86. 